\title{
Case study: Optimization of production processes
}

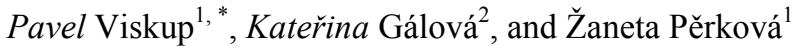 \\ ${ }^{1}$ Tomas Bata University in Zlín, Faculty of Logistics and Crisis Management, Studentské náměstí 1532, 68601 Uherské Hradiště \\ ${ }^{2}$ Tomas Bata University in Zlín, Faculty of Management and Economics, Mostní 5139, 76001 Zlín
}

\begin{abstract}
This article is intended to introduce the reader to the basic ways of increasing the efficiency of current production when problems arise from inaccurate planning of production. The article is based on a study that is gradually implemented as system measures in actual production. Simple and logical improvements bring efficiency improvements and production deadlines. The methods used are suitable for both production and logistics. The study is for a medium-sized company. But the authors want to extend it to small businesses. Small businesses do not fully perceive this area, nor do they accept problems in this area. As part of consultations and inspections, we find that small businesses have significant systemic shortcomings, but owners do not admit this.
\end{abstract}

\section{Introduction}

The main purpose of this study is to approximate shop floor layout optimization and defying time consumption standards in order to ensure timeliness deliveries to customers and improving quality. This case study was processed in the company that is engaged in the development and production of composite and sandwich materials for a wide range of applications in the transport industry sector. The company specializes in demanding applications and specialty products where the need for light and solid materials is very important. The company is engaged in project and serial production. This means production in small series, on demand and customer needs, and assembly line production in large series.

The basic requirement of the company was to propose measures that will ensure an increase in the indicator of fulfilment of delivery deadlines for customers from $57 \%$ to $98 \%$ and reduce the cost caused by rework from $30 \%$ to $15 \%$.

After we were briefly acquainting with the shop floor, it was decided that other activities would focus on optimizing the layout and standardization of work. The development of modern industry represents for shop floor layout planning new challenges. Fast-changing production targets rapidly changing market requirements needs to continuously improve existing shop floor layouts. [1]

Planning and improving facility layout is one of the important issues in production companies because the material handling causes $20-50 \%$ of the total operating costs. [2] Properly set shop floor layout can reduce more than $50 \%$ of operating cost [3]. This effect is caused by cost reduction of activities without added value, for example material handling and transportation or workin-process inventory levels [4].
Traditionally, planning a future shop floor layout is part of the design phase, prior to the construction of the shop floor [5]. Shop floor and facility layout planning has following characteristics: existing facilities may contain critical constraints like stationary machines; layout planning tasks are usually small-scaled like removing and adding machines; used criteria are used ad hoc only for specific tasks. [1]

The effect on productivity, and fulfilment of delivery dates, has as well the work standardization and time consumption standards of individual manufacturing operations. From this point of view, productivity can be increased by reducing work content or by reducing ineffective time for which are used work-studies. Workstudies include method study - finding the best way or "best practice"; and time study - finding the optimal time for every operation [6].

Over the years, different analytical methods for measuring and quantifying employers' manual work have been described and used. All of these methods have only one in common - time as a specific unit. [7] Work measurement concepts can operate with actual measured data or with planned data. Observation methods like time studies or multi-moment analysis are used for finding real times of measured activities. Calculated analytical methods like predetermined time standards (MOST) or simulations then operate with planned data. [8]

For the purpose of this study, we used the time studies and direct measurement of selected activities.

\section{Production layout optimization}

When solving the shop floor arrangement, we proceed by first analysing the current state of workplace layout and production processes and then creating a model of the current state with material flows. There may be more

* Corresponding author: viskup@utb.cz 
suggestions for workplace optimization, and therefore we evaluate proposals for variations in spatial layout. The main purpose of the layout analysis is to identify the current bottlenecks of the manufacturing system and find effective measures that eliminate them.

\subsection{Default state analysis}

The original layout of the workplace was very unsatisfactory at first sight. There was a disorder in the workplace, the working tools had no fixed place and workplace was unsatisfactory in terms of work safety. Workers spent much of the day looking for something, and there was not enough space to work. A big problem was the storage of moulds (e.g. 1,5x2 m dimensions) and handling them in the around the industrial furnace. The industrial furnace has a limited capacity and has become a bottleneck, causing the products and semi-finished products accumulation around. The furnace moulds are left all around the workplace after being pulled out of the oven according to currently empty space.

There were also elements in the layout (Figure 1.) that had to be kept. These elements included the abovementioned furnace and space for other production.

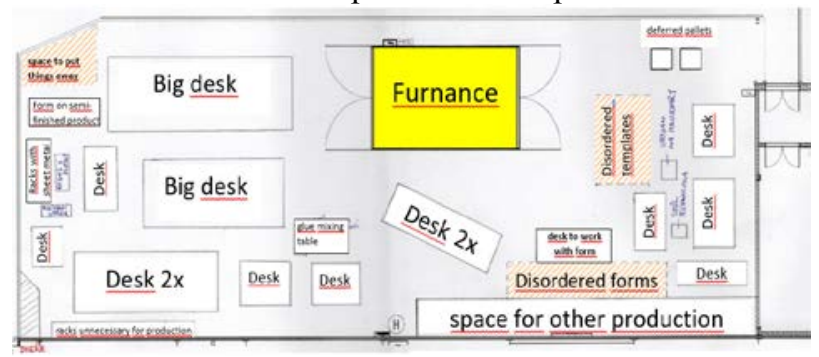

Fig. 1. Original layout [Author].

\subsection{Proposed layout}

Microsoft Visio was used to propose a new layout. The whole workplace was measured before the proposal, so the real scale was considered when working on the proposals. Also, all the necessary workbenches, molds, shelves, etc. were measured. The area of the entire intended workplace is $418 \mathrm{~m}^{2}$.

The new layout proposal was differed from the original as follows:

- Unnecessary workbenches have been removed throughout the workplace, where we found out that they are used in most cases only to postpone things that are no longer needed,

- Fixed place for storing molds, that were previously freely stored in the workshop, was defined, this space will then be standardized,

- There has been a general arrangement of workbenches

- Some workbenches (especially single-purpose) were replaced with multipurpose new ones

- The unnecessary shelves have been removed from the workplace.

- If possible, the needed shelves were replaced by wallmounted shelves.

- The passage of escape routes and the availability of fire extinguishers have been ensured.
- The free space on the walls has been used as a storage for material for production by means of suspension racks.

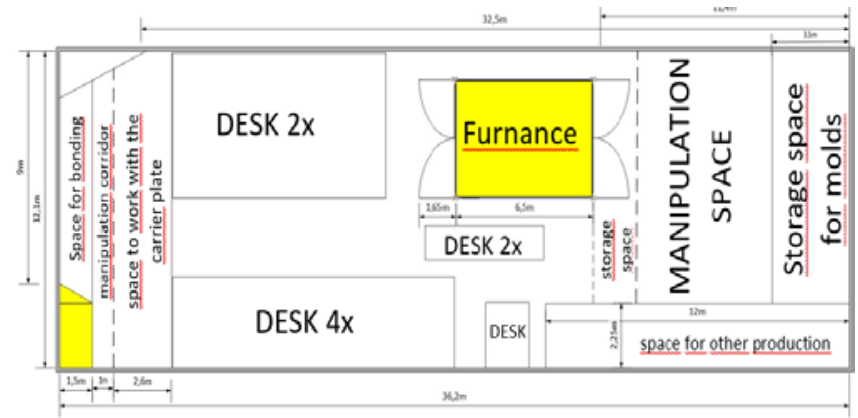

Fig. 2. New layout design [Author].

\section{Time consumption optimization}

Second part of optimization the production processes was time consumption optimization. At this stage, work measurement time study was unnecessary to analyse time consumption and comparing measured times to manufacturing times proposed by firms' technologists.

\subsection{Time study results}

Every worker involved in production process of the products needed for this study was measured. The workers were familiar with the measurement in advance, but they were not told, which employee was observed on particularly day.

Some of basic steps of work measurement time study was followed:

- We used the basic working aids (stationery, form and stopwatch)

- Before the actual measurement, the general activities performed by the workers was described and analyzed

- Measurement started at the beginning of the shift, and the stopwatch run for the entire shift.

- Any activity performed by workers during their shift has been recorded into the form along with the measured time,

- After mapping all work activities, the paper form of the data was transferred to a computerized form using tables in Microsoft Office Excel,

- From the tables, the corresponding graphs were created.

During the observation, an average of fifty activities were recorded for each worker. It should be noted that the activities were performed on the same or similar parts. Since the activities were often repeated, they were reduced to individual groups.

For the purposes of this work, the time studies of four female workers were used. Their work operations differed only minimally, so the results of these studies could be compared and evaluated. Work activities were divided into seven employee activities. The total measured times are stated after the total appreciation in percent (Table 1). 
Table 1. Activities in percentage.

\begin{tabular}{|l|r|r|r|r|}
\hline & \multicolumn{4}{|c|}{ Workers } \\
\hline Activity & \multicolumn{1}{|c|}{ A } & \multicolumn{1}{|c|}{ B } & \multicolumn{1}{c|}{ C } & \multicolumn{1}{c|}{ D } \\
\hline Form cleaning and preparing & $28 \%$ & $4 \%$ & $13 \%$ & $15 \%$ \\
\hline Work with prepreg & $30 \%$ & $18 \%$ & $16 \%$ & $19 \%$ \\
\hline Work with foam & $4 \%$ & $21 \%$ & $18 \%$ & $8 \%$ \\
\hline Form packaging & $10 \%$ & $13 \%$ & $9 \%$ & $14 \%$ \\
\hline Vacuuming & $9 \%$ & $5 \%$ & $3 \%$ & $5 \%$ \\
\hline Cleaning the workplace & $3 \%$ & $4 \%$ & $11 \%$ & $15 \%$ \\
\hline Out of workplace & $13 \%$ & $17 \%$ & $23 \%$ & $16 \%$ \\
\hline
\end{tabular}

It was therefore appropriate to find out the cause of the difference in time for all the studied groups.

A difference of $24 \%$ was found in the form cleaning and preparation work. After reviewing the detailed list of activities, it was found that worker A was looking for the necessary inserts for a relatively long time to construct the part.

A great difference in working times with prepreg was a worker's fault. She did not read the production protocol thoroughly and consequently there was inaccurate measurement and cutting of the prepreg. This, of course, was related to the activity of gluing prepreg, which did not fit into the assembled construction of the sheets and had to be lengthy cut to measure.

The last big difference in time was in the work with industrial foam, where now, compared to other activities, the worker spent the shortest time. The difference between the shortest and the longest measured time was $17 \%$. It was found that in this activity for workers B and $\mathrm{C}$, there was a problem with the misapplied types of foams. It was necessary to find the foam properly or to adjust it to match the production protocol.

Each part has its own identification number, which it also serves for marking technological process, the technical drawings, the times of individual activities and other necessary data.

The corresponding protocol was read prior to the measurement. It lists the individual activities and their times. In the process, the individual activities were divided into groups, each of which contained further activities.

After measuring all parts of the study, all data was processed into the already mentioned Microsoft Office Excel tables. It has been found that almost no measured times correspond to those reported in manufacturing processes, although these times were designed by the company's technology department. Only a minimum of observation times of the parts matched the production protocols.

The table (Table 6) is one example of time comparison, the preparation and folding times were compared. The time difference for part E was just over three hours. It should be noted that this difference is not a mistake of the workers, but after analyzing these activities, a mistake was found rather with the times given by the technology. It has been recognized after consultation with competent persons that the activities being measured are not feasible at specified times.
Table 2. Measured activity time.

\begin{tabular}{|l|c|c|}
\hline \multicolumn{2}{|l|}{$\begin{array}{l}\text { Time from technological } \\
\text { department }\end{array}$} & Measured time \\
\hline \multicolumn{1}{|c|}{ Activity } & Time & Real time \\
\hline Preparing & $0: 10: 00$ & $1: 28: 13$ \\
\hline Folding & $0: 30: 00$ & $2: 13: 52$ \\
\hline Hardening & $3: 30: 00$ & $3: 30: 00$ \\
\hline Finishing & $0: 05: 00$ & $0: 05: 00$ \\
\hline $\begin{array}{l}\text { Cementing, } \\
\text { grinding }\end{array}$ & $0: 15: 00$ & $0: 15: 00$ \\
\hline Control & $0: 02: 00$ & $0: 02: 00$ \\
\hline$\ldots$ another not measured activities \\
\hline Total & $17: 15: 00$ & $20: 17: 05$ \\
\hline Difference & \multicolumn{2}{|c|}{$3: 02: 05$} \\
\hline
\end{tabular}

\subsection{Time standards}

Time standards can be expressed, for example, as time per unit (min / pc) or also quantity per time unit (pc/ hour). The activities covered by the standard are manual activity time, operating time and break time.

The following formula was used for specific calculations: $\mathrm{T}=(\mathrm{OT} * \mathrm{RF})+(\mathrm{OT} * \mathrm{RF} * \mathrm{PFD})$, when:

- OT - Observed Time

- RF - Rating Factor

- PDF Allowance [9]

In addition to the measured time, the degree of performance of workers was determined by $84 \%$ of observations in direct measurements. The surcharge, which is divided into three categories for personal needs, rest and short-term delays, has been consulted with the production manager and industrial engineer. Based on the consultation, the following values were determined:

- surcharges for personal use - $12 \%$

- surcharges - $8 \%$

- surcharges for short-term delays - 5\%

The following table (Tab. 3) was created after calculating the standards according to the formula. The measured parts are categorized by type. As a result, a table of type A is given, the table shows the part number, the original measured time, and the specified standard. The differences between the measured time and the calculated standard are in the order of tens of minutes.

Table 3. Calculated standard times.

\begin{tabular}{|c|c|c|}
\hline $\begin{array}{c}\text { Parts of type } \\
\text { A }\end{array}$ & $\begin{array}{c}\text { Measured time } \\
\text { [minutes] }\end{array}$ & $\begin{array}{c}\text { Calculated standard } \\
\text { time [minutes] }\end{array}$ \\
\hline 1 & 250 & 236 \\
\hline 2 & 390 & 410 \\
\hline 3 & 360 & 378 \\
\hline 4 & 400 & 420 \\
\hline 5 & 460 & 483 \\
\hline 6 & 400 & 420 \\
\hline
\end{tabular}

Time consumption standards has been implemented immediately. Standards have been used in the production planning system. This application of standards has an obvious impact on the right delivery plan, which will not be delayed. However, it is necessary to adjust the set standards in production processes. 


\section{Summary}

Optimizing the shop floor layout and defying the time consumption standards was the main focus of this contribution. However, during the analysis and measurement several shortcomings were observed which decrease work efficiency.

For example, the number of workshop handcart. Each worker should have mobile trolley, which can be manipulated as needed. This trolley should be used by a to lay down work aids such as a tool case, a hot air gun, or personal belongings. The shortage of these trucks was due to new employees. In order to remedy this, an immediate request was made and these trucks were immediately manufactured.

Another drawback was the butyl pistol, this pistol serves for final sealing of the mould. It was found that this pistol is only available to three different manufacturing workshops. Therefore, it was constantly borrowed and traced where the pistol is. Of course, this resulted in undesirable downtime in activities. This issue has been resolved by immediate ordering and delivering additional guns to the workplace.

There was also a lack of the necessary work aids, such as a hot air gun, and each of the workers should have their own and not be looking for and borrowing it somewhere. Again, they were ordered and delivered to the workplace.

Modification of manufacturing processes is ongoing and consists in correcting erroneous time consumption standards.

\section{Conclusion}

To meet the main objective, by the end of 2019 , production deadlines have been raised from $57 \%$ to $98 \%$ and the cost of additional work reduced from $30 \%$ to $15 \%$. Important steps have already been taken. Direct measurement methods were used in the analysis and measurement phase, which consisted of scanning of female workers and individual parts. Furthermore, the layout of the workplace and the data from the measurements were distorted at this stage. Time consumption standards have been set for the improvement phase and a new workplace layout has been proposed. Also, all observed deficiencies were removed.

The checking phase is now in the solution, but it is assumed that the 5S standards are set up and that audits are carried out.

With regard to the current situation in the company, production planning has already improved and so far, the target fulfilment percentage increase from $57 \%$ to $74 \%$ has been fulfilled.

Verifying the goal of working to reduce the cost of extra work is currently in solution. Further long-term imaging is still in progress. So far, the achievement of this goal cannot be assessed, but it cannot be considered an error as the study is still running and there are likely to be some adjustments.
This research was supported by support research program of Tomas Bata University in Zlín (RVO). Funding was extended through: TBU in Zlín No. IGA/FaME/2019/007/ Increasing Business Competitiveness by Meeting Customer Requirements in Customer Relationship Management Using Industrial Engineering Tools.

\section{References}

1. L.N. Pattanaik, B.P. Sharma. The Inter. J o A M T. 42, 7 (2008)

2. G. Wang, Y. Yan, X. Zhang, J. Shangguan, Y. Xiao. IEEE. A simulation optimization approach for facility layout problem. (2008)

3. W. Xie, N.V. Sahinidis. $C \& C E$. 32, 4 (2008)

4. M. Rajasekharan, B.A. Peters, T. Yang. $I J o P R$. 36, 1 (1998)

5. S. Jiang, A.Y.C. Nee. CIRP Annals. 62, 1 (2013)

6. P.V. Chandra. I J o I R $i S, E$ and T. 2, 8 (2013)

7. A. Matias. Work measurement: principles and techniques. (Wiley, New York, 2007)

8. S. Seifermann, J. Böllhoff, J. Metternich, A. Bellaghnach. Conference on Manufacturing Systems proceedings. 588-593. (2014)

9. S. Lawrence, Work measurement and methods improvement. (Wiley, New York, 2000) 